\title{
Knockdown of HMGN2 increases the internalization of Klebsiella pneumoniae by respiratory epithelial cells through the regulation of $\alpha 5 \beta 1$ integrin expression
}

\author{
XINYUAN WANG, JINGYU LI, SHANZE CHEN, XIAOFEI SHEN, XIAOLONG YANG, YAN TENG, \\ LUXIA DENG, YI WANG, JUNLI CHEN, XIAOYING WANG and NING HUANG \\ Department of Pathophysiology, Research Unit of Infection and Immunity, \\ West China College of Basic and Forensic Medicine, Sichuan University, Chengdu, Sichuan 610041, P.R. China
}

Received February 14, 2015; Accepted July 11, 2016

DOI: $10.3892 / \mathrm{ijmm} .2016 .2690$

\begin{abstract}
Integrin receptors, a large family of adhesion receptors, are involved in the attachment of Klebsiella pneumoniae to respiratory epithelial cells, and subsequently cause the internalization of $K$. pneumoniae by host cells. Although a number of molecules have been reported to regulate the expression and activity of integrin receptors in respiratory epithelial cells, the specific underlying molecular mechanisms remain largely unknown. High mobility group nucleosomal binding domain 2 (HMGN2), a non-histone nuclear protein, is present in eukaryotic cells as a ubiquitous nuclear protein. Our previous studies have demonstrated that HMGN2 affects chromatin function and modulates the expression of antibacterial peptide in A549 cells exposed to lipopolysaccharide, which indicates the critical role of HMGN2 in innate immune responses. In addition, our cDNA microarray analysis suggested that HMGN2 knockdown induced the enhanced expression of $\alpha 5 \beta 1$ integrin in A549 cells. Therefore, we hypothesized that intercellular HMGN2 may mediate the internalization of K. pneumoniae by altering the expression of $\alpha 5 \beta 1$ integrin. Using the A549 cell line, we demonstrated that HMGN2 knockdown induced the increased expression of $\alpha 5 \beta 1$ integrin on cell membranes, which resulted in a significant increase in K. pneumoniae internalization. Further results revealed that HMGN2 silencing induced the expression of talin and the activation of $\alpha 5 \beta 1$ integrin, which led to actin polymerization following the phosphorylation of FAK and Src. This study suggests a possible therapeutic
\end{abstract}

Correspondence to: Dr Xiaoying Wang or Professor Ning Huang, Department of Pathophysiology, Research Unit of Infection and Immunity, West China College of Basic and Forensic Medicine, Sichuan University, 17, 3rd Section of Renmin South Road, Chengdu, Sichuan 610041, P.R. China

E-mail: wangxy1004@163.com

E-mail: huangpanxiao@sina.com

Key words: high mobility group nucleosomal binding domain 2, $\alpha 5 \beta 1$ integrin, respiratory epithelial cell, Klebsiella pneumoniae, internalization application for bacterial internalization by targeting HMGN2 in order to treat $K$. pneumoniae infection.

\section{Introduction}

High mobility group nucleosomal binding domain 2 (HMGN2) is a member of the HMG superfamily of non-histone, chromatin-binding proteins, which may be released into the extracellular fluid under pathological conditions of inflammation or cell damage (1-3). The expression of HMG proteins is developmentally regulated. It has been well documented that intranuclear HMG proteins are major regulators of chromosome architecture and gene transcription. Therefore, HMGN2 has been initially regarded as a general transcriptional activator. However, there is increasing evidence to suggest that HMGN2 has multiple intercellular and extracellular functions. It has been well documented that HMGNs may specifically regulate the expression of glycine transporter 1 (GLY1) (4), the transcription factor heat shock protein (HSP)70 (5) and the estrogen-regulated genes TEF1 and FOS (6). $\beta$-defensin is an important innate immune mediator and we have found previously that endogenous HMGN2 may regulate the lipopolysaccharide (LPS)-induced expression of $\beta$-defensin in respiratory epithelial cells $(7,8)$. Our previous research has shown that HMGN2 may be released by interleukin (IL)-2- and phytohemagglutinin (PHA)-stimulated peripheral blood mononuclear cells (PBMCs) (9). Furthermore, exogenous HMGN2 has also been found to exert antimicrobial effects against bacteria, viruses and fungi. In addition, our previous studies showed that exogenous HMGN2 inhibited the invasion of Klebsiella pneumoniae into bladder epithelial cells $(10,11)$ and respiratory epithelial cells (data unpublished). Therefore, HMGN2 as an HMG protein may play a critical role in the innate immune responses induced by mucosal pathogens.

$K$. pneumoniae infection is one of the most frequent hospital-acquired infections, particularly in elderly and immunocompromised individuals. The respiratory tract is the portal of entry and target organ of K. pneumoniae; the persistent localization of $K$. pneumoniae subsequently leads to severe pulmonary infections second only to Pseudomonas aeruginosa in China (12). The integrins are a large family of $\alpha \beta$ heterodimeric transmembrane adhesion receptors that 
mediate cellular interactions with microbes. It has been demonstrated that integrin receptors served as the most important intermediary for the internalization of a series of bacteria by respiratory epithelial cells, including Streptococcus pneumoniae, Staphylococcus aureus, Streptococcus pyogenes and $P$. aeruginosa (13). Therefore, modulating the expression and activity of integrin may interfere with the ability of bacteria to invade host cells. Moreover, our cDNA microarray analysis showed that gene silencing of HMGN2 induced the upregulation of $\alpha 5 \beta 1$ integrin in A549 cells (7). With regard to the multifunctional role of HMGN2 in regulating the expression of genes involved in the specific innate immune response, we aimed to determine whether the silencing of HMGN2 promotes the internalization of $K$. pneumoniae by increasing the expression of $\alpha 5 \beta 1$ integrin in respiratory epithelial cells.

\section{Materials and methods}

Reagents and antibodies. Rabbit anti-human $\alpha 5$ integrin (ab25251) and $\beta 1$ integrin (ab52971) monoclonal antibodies were purchased from Abcam (Cambridge, UK). Talin (T3287) was purchased from Sigma-Aldrich (Shanghai, China). HMGN2 (9437P), phospho-FAK (3284), FAK (3285); phospho-Src (6943) and Src (2109) were purchased from Cell Signaling Technology (Danvers, MA, USA). Rhodamine-conjugated phalloidin, DAPI and FITC were purchased from Sigma-Aldrich. RBITC-conjugated secondary antibody was purchased from Beyotime (Shanghai, China). Cytochalasin B and fibronectin peptide were acquired from Sigma-Aldrich. TRIzol reagent was obtained from Invitrogen (Carlsbad, CA, USA). RevertAid First Strand cDNA Synthesis kit and Maxima ${ }^{\circledR}$ SYBR-Green were obtained from Thermo Fisher Scientific (Vilnius, Lithuania). The PCR primers were obtained from Sangon Biotech Co., Ltd. (Shanghai, China). RPMI-1640 medium was purchased from HyClone, Thermo Scientific (Beijing, China). Fetal bovine serum (FBS) was obtained from FuMeng Gene Co., Ltd. (Shanghai, China). Penicillinstreptomycin was purchased from Beijing Solarbio Science and Technology Co., Ltd. (Beijing, China). Other chemical reagents were all analytical grade.

Strain and cell culture. K. pneumoniae strain 33 was isolated from a sputum sample obtained from a patient with a respiratory infection, which was identified as $K$. pneumoniae by API 20E (bioMérieux, Marcy-l'Étoile, France), at the Medical Department, West China Hospital of Sichuan University (Chengdu, China). Single-colony isolates of $K$. pneumoniae were maintained at $37^{\circ} \mathrm{C}$ on Luria Broth (LB) agar. To infect the epithelial cells, a single colony was grown overnight at $37^{\circ} \mathrm{C}$ in LB medium, and then $50 \mu 1$ of this culture was grown in LB medium for $3 \mathrm{~h}$ until mid-log-phase, washed, and resuspended in phosphate-buffered saline (PBS). The human pulmonary epithelial cell lines A549 and SPC-A-1 as well as the human bronchial epithelial cell line HBE16 from our laboratory were cultured in RPMI-1640 containing 10\% FBS and $1 \%$ penicillin-streptomycin at $37^{\circ} \mathrm{C}$, in $5 \% \mathrm{CO}_{2}$.

Bacterial adhesion and invasion assays. As described in our previous study (10), the A549, SPC-A-1 and HBE16 cells
( $1 \times 10^{5}$ cells/well) were seeded in a 24 -well plate and allowed to adhere overnight. After being washed with PBS, the cells were infected with $K$. pneumoniae strain 33 at a multiplicity of infection (MOI) of 200:1 for $2 \mathrm{~h}$. Non-adherent bacterial cells were removed by washing the cells with PBS. In order to lyse the cells, $200 \mu 10.25 \%$ Triton $\mathrm{X}-100$ was added to each well and incubated at $37^{\circ} \mathrm{C}$ for $10 \mathrm{~min}$. The cells were removed by scraping and plated onto LB agar plates. The colonies were counted to quantify the number of adherent bacteria. For the invasion assay, $100 \mu \mathrm{g} / \mathrm{ml}$ gentamicin (Sichuan Long March Pharmaceutical Co., Ltd., Sichuan, China) was added to kill extracellular bacteria and the cells were incubated at $37^{\circ} \mathrm{C}$ for an additional $2 \mathrm{~h}$. Duplicate samples were included and the experiment was repeated 3 times. We examined the bacterial invasion rate in all three cell lines as well as the time course of invasion by $K$. pneumoniae strain 33 using HMGN2-deficient A549 cells.

RNA interference (RNAi) using small interfering RNA (siRNA) and short hairpin RNA (shRNA) plasmid constructs. The cells were seeded at a density of $5 \times 10^{5}$ cells/well in 6-well plates and allowed to reach $60 \%$ confluence on the day of transfection. The small interfering RNA (siRNA) and shRNA for HMGN2 were synthesized at our laboratory as previously described as well as shRNA control (shControl) and siRNA control (siControl) (7). HMGN2-overexpressing (pexHMGN2) and control (pexControl) vectors were constructed using a pEX-1-HMGN2 vector (GenePharma. Inc, Shanghai, China). shRNA HMGN2, 5'-GCAAAGGTGAAGGACGAACCA-3' or shRNA control, 5'-GCTTCGCGCCGTAGTCTTA-3' were cloned into a psi-LVRH1GP vector (Fulengen. Inc, Guangzhou, China). The cells were transfected for $24 \mathrm{~h}$ with siRNA or shRNA plasmids using Lipofectamine 2000 reagent in Opti-MEM medium.

Reverse transcription-quantitative polymerase chain reaction $(R T-q P C R)$. The effects of siHMGN2 on the expression of the cell integrin gene encoding $\alpha 5 / \beta 1$ integrin, which is critical for the invasion of $K$. pne umoniae into human lung epithelial cells, were investigated in A549 cells by RT-qPCR. Total RNA was extracted using TRIzol reagent. cDNA synthesis was achieved using the RevertAid First Strand cDNA Synthesis kit. The cDNA synthesized served as the template for RT-qPCR amplification of $\alpha 5 / \beta 1$ integrin. The PCR products were detected using Thermo Fisher Scientific Maxima SYBRGreen. The primer sequences were as follows: $\alpha 5$ integrin forward, 5'-TGCAGTGTGAGGCTGTGTACA-3' and reverse, 5'-GTGGCCACCTGACGCTCT-3'; and $\beta 1$ integrin forward, 5'-CTCAAGCCAGAGGATATTAC-3' and reverse, 5'-TCATT GAGTAAGACAGGTCC-3' $(14,15)$. The relative levels of $\alpha 5 /$ $\beta 1$ integrin mRNA transcripts in different groups were evaluated using the $2^{-\Delta \Delta \mathrm{Ct}}$ method.

Western blot analysis. Western blot analysis was performed as previously described (10). The A549 cells were collected and lysed in lysis buffer ( $1 \mathrm{ml}$ containing $5 \mu \mathrm{l}$ PMSF, $10 \mu \mathrm{l}$ phosphatase inhibitors and $1 \mu \mathrm{l}$ protease inhibitors). The cell lysates were centrifuged at $13,400 \mathrm{x}$ g for $20 \mathrm{~min}$ at $4^{\circ} \mathrm{C}$. The total protein concentration was determined using the Bradford assay. Equal amounts of the lysate were added into the wells of the SDS-PAGE gel. The proteins were then transferred 
Table I. List of integrin and associated partial genes with changed expression identified by microarray analysis.

\begin{tabular}{llll}
\hline GenBank & $\begin{array}{c}\text { CGAP gene } \\
\text { symbol }\end{array}$ & $\begin{array}{c}\text { Log2 ratio } \\
\text { CVA }\end{array}$ & \\
\hline NM_014288.3 & ITGB3BP & 0.916241 & Integrin beta 3 binding protein (beta3-endonexin) \\
NM_002214.1 & ITGB8 & 0.9928003 & Integrin beta 8 \\
NM_002211.2 & ITGB1 & 1.042064 & Integrin beta 1 (fibronectin receptor, beta polypeptide, antigen CD29 includes \\
& & & MDF2) \\
NM_002203.2 & ITGA2 & 1.3149265 & Integrin alpha 2 (CD49B, alpha 2 subunit of VLA-2 receptor) \\
NM_000211.1 & ITGB2 & 1.5798765 & Integrin beta 2 (antigen CD18 (p95), lymphocyte function-associated antigen 1) \\
NM_000889.1 & ITGB7 & 0.437597 & Integrin beta 7 \\
NM_002210.2 & ITGAV & 0.712578 & Integrin alpha V (vitronectin receptor, alpha polypeptide, antigen CD51) \\
AH008066.2 & ITGA6 & 4.42582719 & Integrin alpha 6 \\
NM_002207.2 & ITGA9 & 0.7271413 & Integrin alpha 9 \\
NM_004791.2 & ITGBL1 & 0.7609444 & Homo sapiens integrin subunit beta like 1 \\
BU738798 & ILK & 0.2331936 & Integrin-linked kinase \\
NM_003930.3 & SCAP2 & 0.683518 & Src kinase-associated phosphoprotein 2 \\
NM_032214.2 & SLA2 & 0.1621274 & Src-like-adaptor 2 \\
NM_020209.2 & SHD & -1.2045916 & Src homology 2 domain containing transforming protein D \\
NM_000261.1 & MYOC & 2.2054772 & Myocilin, trabecular meshwork inducible glucocorticoid response \\
NM_023940.2 & RASL11B & 2.3479829 & RAS-like, family 11, member B \\
NM_005406.1 & ROCK1 & -2.461461 & Rho-associated, coiled-coil containing protein kinase 1 \\
NM_004333.2 & BRAF & -2.31846 & v-raf murine sarcoma viral oncogene homolog B1 \\
BC038293 & PTENP1 & -1.166008 & Phosphatase and tensin homolog (mutated in multiple advanced cancers 1), \\
NM_022748.6 & TENS1 & -1.0666966 & pseudogene 1 \\
Tensin-like SH2 domain containing 1 \\
NM_006289.2 & TLN1 & -0.527023 & Talin 1 \\
\hline
\end{tabular}

onto a PVDF membrane and blocked with $5 \%$ non-fat milk in Tris-buffered saline solution for $1 \mathrm{~h}$, and incubated with the primary antibodies overnight at $4^{\circ} \mathrm{C}$. The membrane was incubated with the secondary antibody $(1: 1,000)$ for $2 \mathrm{~h}$ at room temperature. The intensity of the bands was quantified relative to corresponding GAPDH bands with the Bio-Rad VersaDoc $^{\mathrm{TM}}$ Imaging system (Berkeley, CA, USA).

Flow cytometric analysis. The A549 or HBE16 cells were infected with $K$. pneumoniae pre-treated with siRNA HMGN2 or siRNA control at an MOI of 200:1 for $1 \mathrm{~h}$ at $37^{\circ} \mathrm{C}$. The cells were then washed vigorously, detected, fixed with $4 \%$ paraformaldehyde and permeabilized with $0.1 \%$ Triton X-100. The cells were stained with rhodamine-conjugated phalloidin for $60 \mathrm{~min}$. For the $\alpha 5 / \beta 1$ integrin assay, the cells were incubated with the rabbit monoclonal antibodies at $4^{\circ} \mathrm{C}$ overnight, and then incubated with anti-rabbit secondary antibody for $1 \mathrm{~h}$. Flow cytometric analysis was performed using a BD Accuri ${ }^{\mathrm{TM}}$ C6 flow cytometer (BD Biosciences, Franklin Lakes, NJ, USA) and repeated thrice.

Immunofluorescence microscopy. The A549 cells were washed thrice in PBS, and subsequently fixed with $4 \%$ paraformaldehyde for $30 \mathrm{~min}$. The cells were then permeabilized for $10 \mathrm{~min}$ in PBS containing $0.1 \%$ Triton X-100, and washed 3 times in PBS. The cells were stained with rhodamine-conjugated phalloidin for $1 \mathrm{~h}$ to visualize filamentous actin (F-actin), and the cell nuclei were stained with 4',6-diamidino-2-phenylindole (DAPI) for $5 \mathrm{~min}$. The bacteria were stained with FITC for $1 \mathrm{~h}$. For the $\alpha 5 / \beta 1$ integrin stain, the cells were incubated with the rabbit monoclonal antibodies at $4^{\circ} \mathrm{C}$ overnight, then recovered at $37^{\circ} \mathrm{C}$ for $30 \mathrm{~min}$ and incubated with anti-rabbit secondary antibody at $37^{\circ} \mathrm{C}$ for $30 \mathrm{~min}$. The stained cells were observed under an immunofluorescence microscope (VMF30A; Carl Zeiss, Oberkochen, Germany).

Cell adhesion assay. A cell adhesion assay was performed as previously described by Docheva et al (16). Briefly, a 96-well plate was coated with $50 \mu \mathrm{g} / \mathrm{ml}$ fibronectin overnight at $4^{\circ} \mathrm{C}$ and then blocked with $1 \%$ bovine serum albumin (BSA) powder in PBS for $1 \mathrm{~h}$ at $37^{\circ} \mathrm{C}$. The A549 cells $\left(3 \times 10^{4}\right.$ cells/well) were plated and incubated for $2 \mathrm{~h}$ at $37^{\circ} \mathrm{C}$. Non-adherent cells were removed by washing with PBS. The number of adherent cells was estimated using a cell counting kit-8 (CCK-8; purchased from Vazyme Biotech, Shanghai, China) and the absorbance at $405 \mathrm{~nm}$ was measured by a Bio-Rad iMark ${ }^{\mathrm{TM}}$ microplate reader. The percentage of adherent cells on fibronectin was calculated to a maximal reference. Three independent experiments were performed in triplicate.

Statistical analysis. The two-tailed Student's t-test was used to assess the differences in values between the experimental groups. A P-value $<0.05$ was considered to indicate a statistically significant difference. 


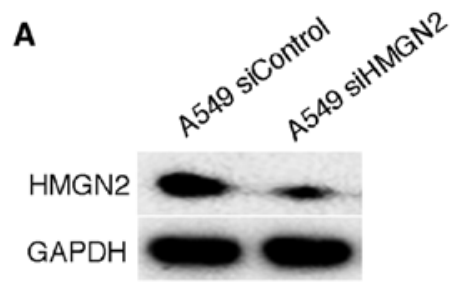

B

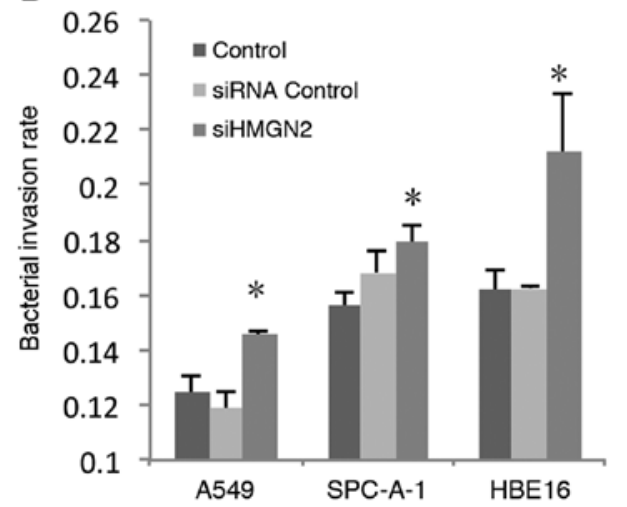

C

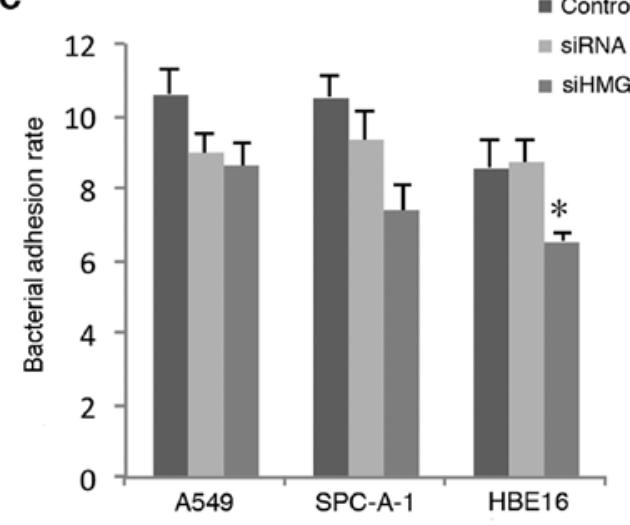

Figure 1. Effects of downregulation of high mobility group nucleosomal binding domain 2 (HMGN2) expression on K. pneumoniae strain 33 adhesion to and internalization by respiratory epithelial cells. (A) The cells were transfected with HMGN2 siRNA or control siRNA. Twenty-four hours later, the whole cell lysates were then subjected to western blot analysis. (B) A549, SPC-A-1 and HBE16 cells were transfected with HMGN2 siRNA or control siRNA, and Lipofectamine 2000 for $24 \mathrm{~h}$, then infected with $K$. pneumoniae strain 33 at an MOI of 200:1 for $2 \mathrm{~h}$. Incubation with gentamicin (100 $\mu \mathrm{g} / \mathrm{ml})$ for $2 \mathrm{~h}$ eliminated extracellular bacteria. The numbers of invading bacteria were counted on the LB viable plate. Invasion rate, invasion bacteria number/cell number. (C) A549, SPC-A-1 and HBE16 cells were transfected with HMGN2 siRNA or control siRNA, and Lipofectamine 2000 for 24 h, and then infected with K. pneumoniae strain 33 at an MOI of 200:1 for $2 \mathrm{~h}$. Adhesion rate, adhesion bacteria number/cell number. The data are expressed as the means \pm SEM of three individual experiments. ${ }^{*} \mathrm{P}<0.05$ vs. siRNA control.

\section{Results}

Gene expression profiles associated with integrin in HMGN2-silenced A549 cells. To explore the role of HMGN2 in the regulation of gene transcription in respiratory epithelial cells, we employed siRNA targeted against HMGN2 in A549 cells. In our previous study, we used a cDNA microarray to test approximately 31,000 genes; the expression of $>4 \%$ of the genes was changed by $\geq 2$-fold in the HMGN2-silenced A549 cells (7). The results of cDNA microarray analysis revealed that disruption of HMGN2 induced the expression of 10 integrin subunits and a series of related genes changed. Specifically, integrin alpha 5 gene (ITGA5) was increased 1.64-fold and integrin beta 1 gene (ITGB1) was upregulated 2.06-fold in the A549 cells depleted of HMGN2. Furthermore, we found that the integrin-activated protein gene integrin-linked kinase (ILK) was upregulated and the integrin activity inhibiting proteins PTENP1 and TENS1 were downregulated. In addition, Src kinase-associated phosphoprotein 2 (SCAP2) gene was increased 1.61-fold compared with the siRNA control A549 cells (Table I). These changes in gene expression profiles are associated with cytoskeletal rearrangement, cell adhesion, migration, proliferation and mechanical sensing.

HMGN2 gene knockdown increases the internalization of $K$. pneumoniae strain 33 by pulmonary and bronchial epithelial cells. HMGN2 has been suggested to significantly inhibit the internalization of $K$. pneumoniae by bladder epithelial cells (10). In addition, HMGN2 may generally or specifically modulate gene expression. Herein, we examined whether disrupting the expression of HMGN2 affected the ability of the host cell to interact with bacteria. We used siRNA to silence the expression of HMGN2 in the following cell lines: A549 (Fig. 1A); SPC-A-1 and HBE16 (data not shown).

$K$. pneumoniae internalization by the epithelial cells was examined using a bacterial invasion assay. The cells were co-incubated with K. pneumoniae strain 33 at an MOI of 200:1 for $2 \mathrm{~h}$, and the bacterial adhesion and invasion rates were detected, respectively. The results showed that the pulmonary epithelial cells (A549 and SPC-A-1) and the bronchial epithelial cells (HBE16) depleted of HMGN2 demonstrated enhanced internalization of $K$. pneumoniae strain 33 when compared with the controls (Fig. 1B). The results indicate that depleted HMGN2 induced the internalization of K. pneumoniae strain 33 by respiratory epithelial cells. However, the adhesion of $K$. pneumoniae strain 33 to HBE16 cells was decreased (Fig. 1C).

Invasion kinetics of K. pneumoniae strain 33 in A549 cells transfected with HMGN2 RNAi. In order to further explore bacterial internalization by respiratory epithelial cells, we examined the time course of invasion by $K$. pneumoniae strain 33 using HMGN2 RNAi in A549 cells. The A549 cells were infected with $K$. pneumoniae strain 33 at an MOI of 200:1, and the numbers of intracellular K. pneumoniae bacteria were quantified at various time intervals (Fig. 2A). Bacterial internalization by HMGN2-deficient A549 epithelial cells was completed after a 1-h incubation. The bacterial invasion rate increased in a time-dependent manner, and the rate in the HMGN2 siRNA group was always higher in comparison with that in the control group at each time point. In this assay, at $6 \mathrm{~h}$ a bacterial invasion rate of $0.405 \pm 0.035$ bacteria per cell 


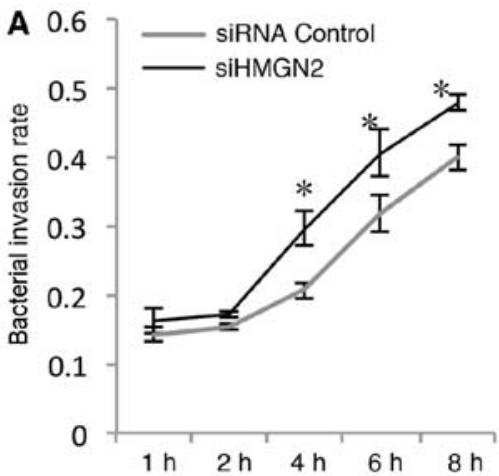

B

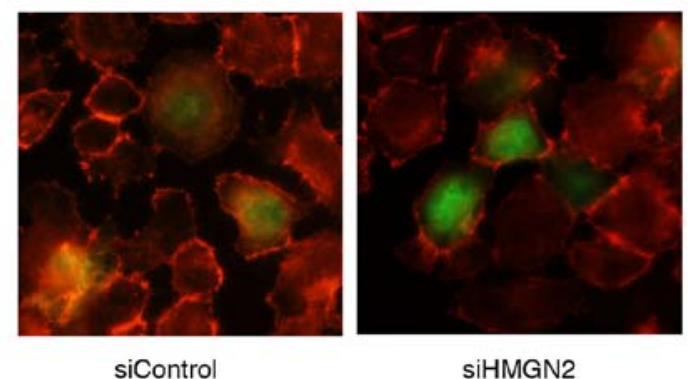

Figure 2. Effects of silencing high mobility group nucleosomal binding domain 2 (HMGN2) on the invasion kinetics of $K$. pneumoniae strain 33 into A549 cells. (A) A549 cells were transfected with HMGN2 siRNA or control siRNA for $24 \mathrm{~h}$, and then infected with K. pneumoniae strain 33 at an MOI of $200: 1$ for the indicated time periods. Incubation with gentamicin $(100 \mu \mathrm{g} / \mathrm{ml})$ for $2 \mathrm{~h}$ eliminated extracellular bacteria. Invasion rate, invasion bacteria number/cell number. (B) A549 cells transfected with HMGN2 siRNA or control siRNA then infected with K. pneumoniae strain 33. The cells were labeled with rhodamine-conjugated phalloidin (red) and bacteria were labeled with FITC (green). siRNA control A549 cells infected with K. pneumoniae (left panel, siControl); HMGN2 siRNA A549 cells infected with $K$. pneumoniae (right panel, siHMGN2). The data are expressed as the means \pm SEM of three individual experiments ${ }^{*} \mathrm{P}<0.05$ vs. siRNA control.

was detected in the HMGN2 siRNA group, which was 2.5-fold higher than the bacterial invasion rate at $1 \mathrm{~h}$.

To further confirm the increased intracellular presence of bacteria by silencing HMGN2 in the cells, HMGN2-silenced A549 monolayers were infected with FITC-labeled $K$. pneumoniae strain 33 at an MOI of $200: 1$ at $37^{\circ} \mathrm{C}$ for $2 \mathrm{~h}$. We showed that the bacteria interacted with the cells using immunofluorescence microscopy (Fig. 2B).

Silencing HMGN2 mediates the upregulation of $\alpha 5 \beta 1$ integrin on cell membranes. To elucidate the mechanism responsible for the induction of bacterial internalization by HMGN2-silenced A549 cells, we measured $\alpha 5 \beta 1$ integrin mRNA levels in the HMGN2-silenced A549 cells. Silencing HMGN2 in the A549 cells increased the mRNA expression of $\beta 1$ integrin, whereas it decreased the mRNA expression of $\alpha 5$ integrin (Fig. 3A). We used shRNA to silence the expression of HMGN2 and an overexpression plasmid to overexpress HMGN2 in A549 cells (Fig. 3B). To explore the functions of integrins on the cell membrane, we examined the expression of $\alpha 5 \beta 1$ integrin on the surface of A549 cells using flow cytometry. $\alpha 5$ and $\beta 1$ integrin proteins located on the cell surface were also studied by employing an RBITC-conjugated secondary antibody, which emits green fluorescence (FL1-positive; green). The expression of $\alpha 5$ integrin was upregulated in the HMGN2-deficient A549 cells both by siRNA and shRNA silencing, and the expression of $\beta 1$ integrin was also higher when compared with the siRNA control A549 cells (Fig. 3C and D). Overexpression of HMGN2 downregulated $\alpha 5$ integrin and $\beta 1$ integrin protein expression on the cell membrane. In addition, $\alpha 5 / \beta 1$ integrin was detected on the cell membrane by fluorescence microscopy (Fig. 3E).

Talin is involved in silencing HMGN2-induced $\alpha 5 \beta 1$ integrin activity. Integrin activity is a key factor which determines the host cell interaction with pathogens. Integrin activation is determined by the conformation of $\alpha$ and $\beta$ subunits on the cell membrane, which are regulated by inside-out activation (17). Having established that silencing HMGN2 induces the internalization of K. pneumoniae by respiratory epithelial cells, we next examined whether it is due to effects on $\alpha 5 \beta 1$ integrin activity. Fibronectin is known to be an important integrin ligand existing in the extracellular matrix (ECM). Therefore, we examined the ability of A549 cells to adhere to fibronectin. The cell adhesion assay indicated that HMGN2 knockdown in the A549 cells induced the adhesion of $\alpha 5 \beta 1$ integrin to fibronectin (Fig. 4A). It has been reported that talin plays an important role in $\alpha 5 \beta 1$ integrin activation (18). To examine the role of talin in the HMGN2-mediated regulation of $\alpha 5 \beta 1$ integrin expression and activity, western blot analysis was used to examine the protein levels of talin. The results showed that the transfection of cells with HMGN2 siRNA or shRNA increased talin expression, and the overexpression of HMGN2 in A549 cells suppressed talin expression (Fig. 4B).

FAK/Src signaling pathway is involved in silencing HMGN2 in A549 cells. FAK/Src may be recruited to cell membranes and phosphorylated by activated $\alpha 5 \beta 1$ integrin. We examined whether silencing HMGN2 enhanced FAK/Src activation. As shown in Fig. 5, knockdown of HMGN2 using shRNA induced the phosphorylation of FAK and Src by $>20$ and 25\%, respectively. The overexpression of HMGN2 in the A549 cells decreased the phosphorylation levels of FAK and Src. These data indicate that silencing HMGN2 in A549 cells induces the activation of FAK and Src.

Knockdown of endogenous HMGN2 induces actin cytoskeleton reorganization. Microfilament polymerization is an important process for most bacteria entering eukaryotic cells. Our previous studies $(10,11)$ revealed that $K$. pneumoniae infection induces actin polymerization. To examine the polymerization of actin, respiratory epithelial cells were transfected with HMGN2 siRNA for $24 \mathrm{~h}$, and as a positive control, the cells were pre-treated with $10 \mu \mathrm{g} / \mathrm{ml}$ cytochalasin $\mathrm{B}$, an inhibitor of actin rearrangement. The cells were then incubated with $K$. pneumoniae stain 33 for $2 \mathrm{~h}$ at $37^{\circ} \mathrm{C}$, and analyzed using flow cytometry. As shown in Fig. 6A and B, K. pneumoniae 33 infection induced actin polymerization and HMGN2-silenced A549 and HBE16 cells showed significantly higher amounts of F-actin. The data indicates that the depletion of HMGN2 

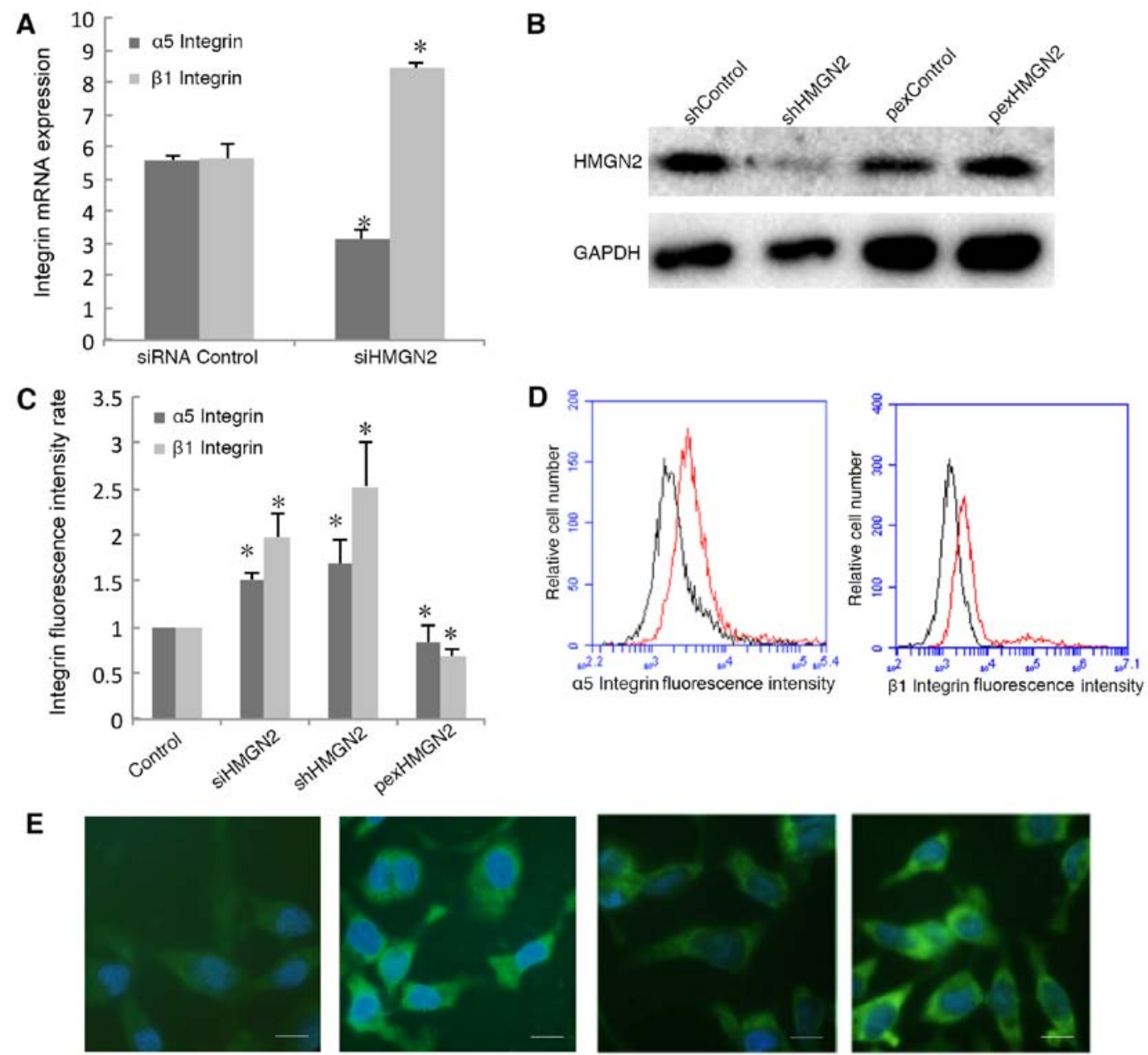

siControl SIHMGN2

siControl SIHMGN2

A549 a5 Integrin A549 $\beta 1$ Integrin

Figure 3. Effects of silencing high mobility group nucleosomal binding domain 2 (HMGN2) on $\alpha 5 \beta 1$ integrin expression. (A) A549 cells transfected with HMGN2 siRNA or control siRNA for 24 h. $\alpha 5$ and $\beta 1$ integrin expression was examined by RT-qPCR. (B) A549 cells were transfected with the control or HMGN2 shRNA, as well as the HMGN2-overexpressing plasmid for $24 \mathrm{~h}$, and later the whole cell lysates were subjected to western blot analysis. (C) A549 cells were transfected with HMGN2 siRNA or control siRNA for $24 \mathrm{~h} . \alpha 5$ and $\beta 1$ integrin expression was examined using flow cytometric analysis. The data are expressed as the means \pm SEM of 3 individual experiments. ${ }^{*}<0.05$ vs. siRNA control. (D) Histograms of $\alpha 5$ and $\beta 1$ integrin expression which were evaluated using flow cytometry. HMGN2 siRNA group (red line) and control siRNA group (black line). (E) $\alpha 5$ and $\beta 1$ integrin on A549 cell membranes were labeled with integrin antibody-RBITC conjugated secondary antibody (green), and the cell nuclei were labeled with DAPI (blue) and observed under a fluorescence microscope. Scale bars represent $10 \mu \mathrm{m} . \alpha 5$ and $\beta 1$ integrin expression was enriched in HMGN2-silenced A549 cells.

promoted the assembly of actin. Morphological evidence was obtained by fluorescence microscopy. Staining of the cells with rhodamin-conjugated phalloidin revealed that well-organized actin filaments were more clear in the HMGN2-silenced A549 cells (Fig. 6C).

\section{Discussion}

The airway epithelium represents a primary site for contact between microbes and their hosts. Entry into epithelial cells is considered to be an important strategy for microorganisms to escape immune clearance (19). Our previous research has suggested that exogenous HMGN2 significantly inhibits the internalization of $K$. pneumoniae by bladder epithelial cells (10). However, the role of endogenous HMGN2 in the regulation of $K$. pneumoniae internalization remains unclear. In the present study, we firstly investigated whether endogenous HMGN2 was involved in the internalization of $K$. pneumoniae by respiratory epithelial cells. We found that the transfection of pulmonary and bronchial cells with HMGN2 siRNA induced the increased internalization of $K$. pneumoniae strain 33; however, the decreased adhesion of $K$. pneumoniae strain 33 to the bronchial epithelial HBE16 cells was observed. The results suggested that endogenous HMGN2 may also be engaged in the epithelial cells to anti-microbial invasion process of the respiratory tract, which is conducive to the innate immune defenses as well as beneficial for the elimination of bacteria.

The adhesion of bacteria to mucosal and epithelial surfaces is often the first stage in host cell invasion. In general, it has been revealed that the adhesion of bacteria to respiratory epithelial cells may promote bacterial cell invasion $(20,21)$. However, we observed that the $K$. pneumoniae adhesion rate was not significantly altered or even reduced following HMGN2 knockdown in respiratory epithelial cells, which 
A

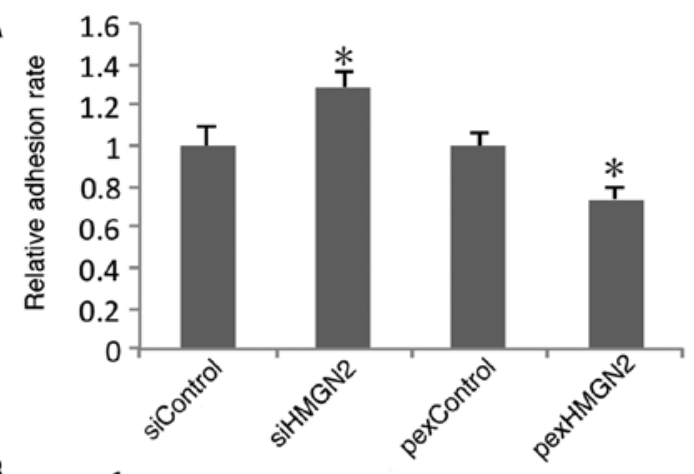

B
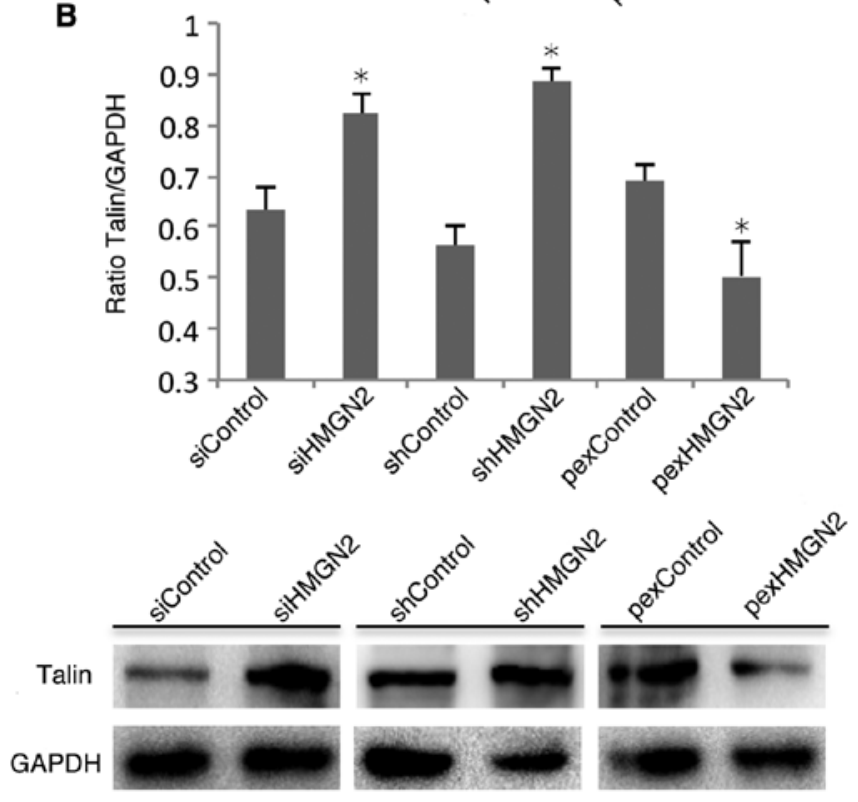

Figure 4. Talin is involved in silencing high mobility group nucleosomal binding domain 2 (HMGN2)-induced $\alpha 5 \beta 1$ integrin activity. (A) A549 cells were transfected with either the controls, HMGN2 siRNA or HMGN2-overexpressing plasmid for $24 \mathrm{~h}$, and subsequently the whole cell adhesion to fibronectin was determined using a microplate reader. (B) A549 cells were transfected with the controls, HMGN2 siRNA, HMGN2 shRNA, or HMGN2-overexpressing plasmid for $24 \mathrm{~h}$. The whole cell lysates were then subjected to western blot analysis. The data are expressed as the means \pm SEM of three individual experiments. ${ }^{*} \mathrm{P}<0.05$ vs. siRNA control.

indicated that the increased $K$. pneumoniae invasion rate induced by HMGN2 knockdown in respiratory epithelial cells is not affected by the adhesion rate.

Adhesion and invasion are both mutually interrelated and independent complex processes. The internalization of bacteria by epithelial cells is not only associated with the adhesion of bacteria to the cell membrane receptors, but it is also associated with the intracellular signals that regulate the cell surface receptors and cytoskeleton polymerization state (22,23). Cue et al (24) have reported that a nonpeptide integrin antagonist may inhibit $S$. pyogenes invasion whereas it increases bacterial adhesion to epithelial cells through blocking $\alpha 5 \beta 1$ integrin. Takagi et al (17) and Vinogradova et al (25) developed the 'inside-out' integrin signaling model, which stated that the intracellular signal may influence the conformation and ligand-binding affinity of the extracellular domain of integrins. This 'inside-out' signal transduction appears to be mediated through the integrin cytoplasmic domains. Our cDNA microarray results showed that $\alpha 5$ and $\beta 1$ integrin were upregulated in the HMGN2-silenced A549 cells (7). Therefore, we hypothesized that the upregulated expression of $\alpha 5 \beta 1$ integrin, induced by HMGN2 knockdown, mediated the increased internalization of K. pneumoniae by A549 cells.

Integrins are cell transmembrane adhesion receptors, which contain at least $18 \alpha$ and $8 \beta$ subunits that form 24 different $\alpha \beta$ integrins in distinct cells (26). These heterodimers on the cell surface have been demonstrated to play a pivotal role in regulating cell-cell, cell-ECM and cell-bacteria interactions to control cell fate in cancer biology and inflammation. $\alpha 5 \beta 1$ integrin, is composed of $\alpha 5$ and $\beta 1$ subunits and it has been described as a receptor which binds to fibronectin as well as having a well-defined role in cell and bacterial adhesion (27). It has been reported that HMGB1, another type of HMG protein, may induce motility in chondrosarcoma cells through upregulating $\alpha 5 \beta 1$ integrin expression (28). Moreover, previous findings have indicated that $\alpha 5 \beta 1$ integrin acts as a membrane receptor to recognize a series of Gram negative and positive pathogens (13). Therefore, we postulated that HMGN2 has a similar function and it regulates $\alpha 5 \beta 1$ integrin expression and subsequently affects cellular behavior.

To determine whether HMGN2 regulates the expression of $\alpha 5 \beta 1$ integrin in respiratory epithelial cells, the RNAi technique was used to disrupt HMGN2 expression in A549 cells. Subsequently, both the mRNA and protein levels of $\alpha 5 \beta 1$ integrin were analyzed. Flow cytometric analysis and immunofluorescence microscopy revealed that the expression of $\alpha 5$ and $\beta 1$ integrin on cell membranes was increased by HMGN2 knockdown in A549 cells. However, RT-qPCR revealed only the increased $\mathrm{mRNA}$ expression of the $\beta 1$ subunit, whereas the mRNA level of $\alpha 5$ integrin was decreased, which differs from the cDNA microarray data (7). For this paradox, there are at least two main reasons. One possibility is that false positive results existed in the microarray analysis; another possible reason is that HMGN2 may regulate the transportation of $\alpha 5 \beta 1$ integrin from the cytoplasm to the cell membrane leading to the $\alpha 5 \beta 1$ integrin distribution change, which possibly explains the decreased presence of $\alpha 5$ integrin on the cell membranes. Johnson et al have demonstrated that integrin $\alpha$ and $\beta$ subunits are combined in the endoplasmic reticulum and transported to the cell surface before functioning as a molecular bridge between cellular 'inside and outside' signaling (29).

The activation of cell surface integrin is determined by integrin $\alpha$ and $\beta$ subunit conformation. The integrin heterodimers have three different affinity phases: inactive; active and ligand occupied states; a dynamic equilibrium exists among them (30). External signal stimulation may break up the salt bridge between the $\alpha$ and $\beta$ subunit and therefore inhibit the activation of integrin. Fibronectin, a $\alpha 5 \beta 1$ integrin ligand, exists in the ECM. An established cell adhesion assay testing the ability of $\alpha 5 \beta 1$ integrin to bind to fibronectin was used to determine the integrin binding activity. Our results showed that silencing HMGN2 in the A549 cells increased the adhesion rate of epithelial cells to fibronectin, which indicates that HMGN2 may play a role in switching integrin active states (Fig. 4A).

Integrin activation is a dynamic, tightly-regulated process, which is regulated by both positive and negative regulators (30). Talins and kindlins, two families of FERM-domain 

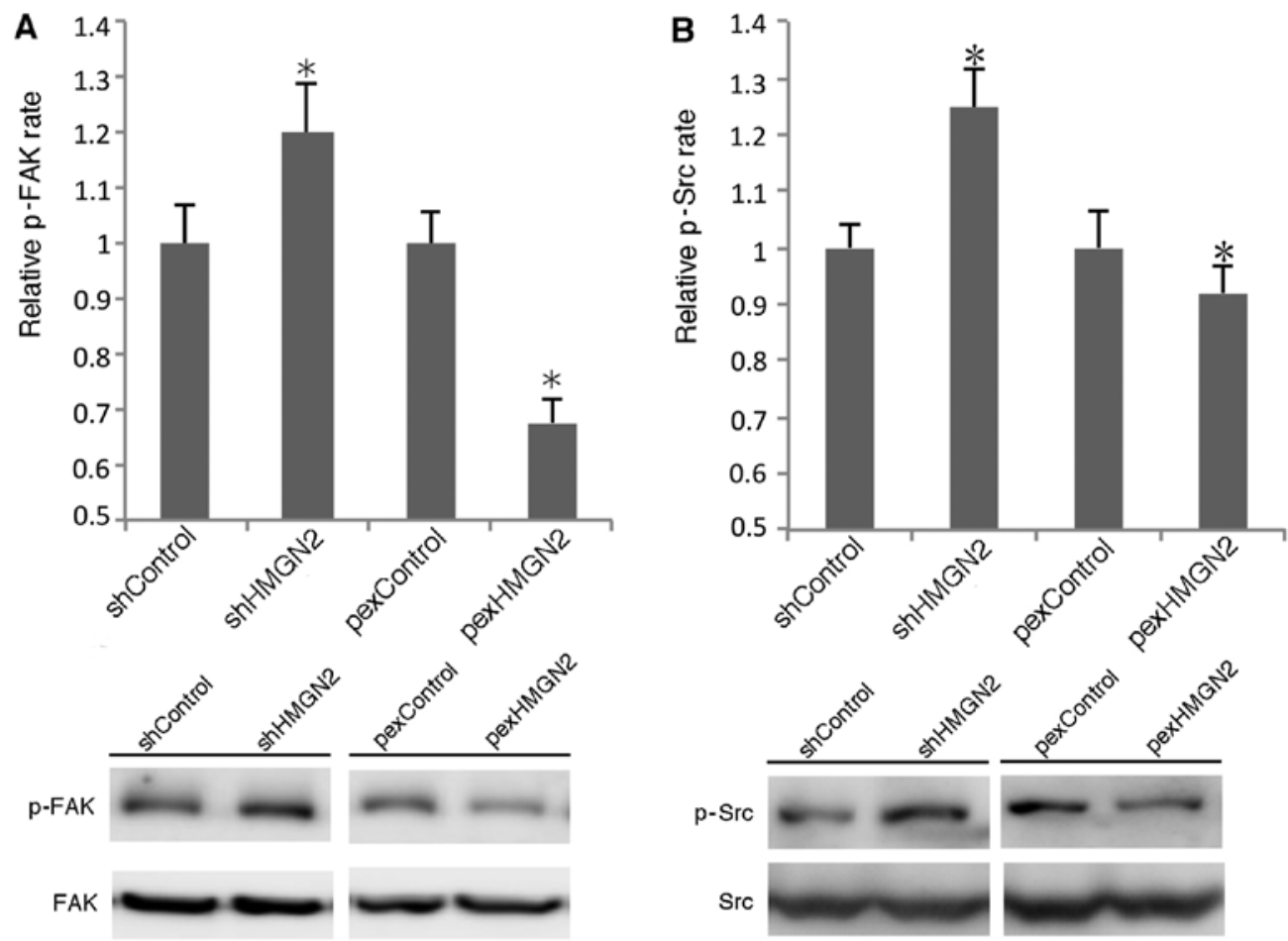

Figure 5. Effects of silencing high mobility group nucleosomal binding domain 2 (HMGN2) on FAK/Src signaling. (A and B) A549 cells were transfected with the control, HMGN2 shRNA, or HMGN2-overexpressing plasmid for $24 \mathrm{~h}$. The whole cell lysates were then subjected to western blot analysis to determine-the phosphorylation levels of FAK and Src. The data are expressed as the means \pm SEM of 3 individual experiments. " $\mathrm{P}<0.05$ vs. control.
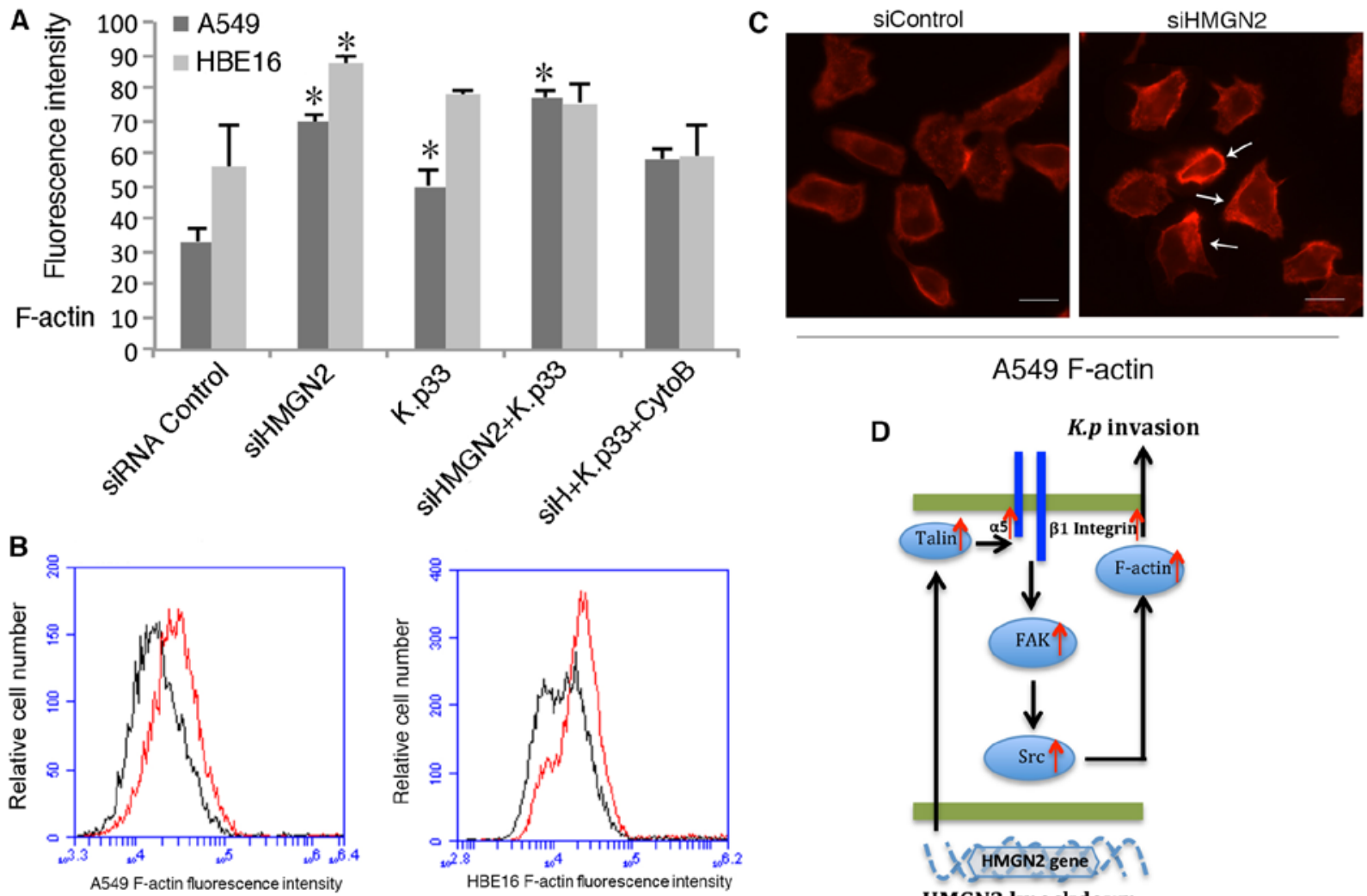

\section{A549 F-actin}

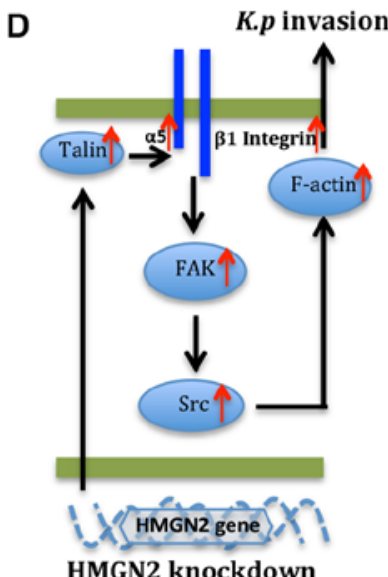

Figure 6. Effects of silencing high mobility group nucleosomal binding domain 2 (HMGN2) on actin polymerization. (A) A549 and HBE-16 cells were transfected with HMGN2 siRNA and control siRNA for $24 \mathrm{~h}$, and then treated with cytochalasin B (CytoB) and Klebsiella pneumoniae strain 33 (K.p33) for $2 \mathrm{~h}$, respectively. The relative F-actin fluorescence intensity was examined by flow cytometry. The data are expressed as the means \pm SEM of 3 individual experiments. "P<0.05 vs. control. (B) Histogram of F-actin expression by flow cytometry. HMGN2 siRNA group (red line) and control siRNA group (black line). (C) A549 cells were labeled with rhodamine-conjugated phalloidin (red) and observed under a fluorescence microscope. Scale bars represent $10 \mu \mathrm{m}$. control siRNA A549 (left panel, siControl); HMGN2 siRNA A549 (right panel. siHMGM2). (D) Schematic representation of the signaling pathways involved in silencing HMGN2-induced K. pneumoniae infection as well $\alpha 5 \beta 1$ integrin expression of respiratory epithelial cells. 
proteins, bind to the cytoplasmic tail of integrins and recruit cytoskeletal and signaling proteins, which have been well documented to positively regulate the activation of integrin (26). It has been demonstrated that talin may loosen the integrin transmembrane coiled-coil structure, which further affects the affinity of integrin for extracellular ligands as well as cytoskeleton polymerization $(18,31)$. We found that silencing HMGN2 upregulated the protein expression of talin, which was decreased by the overexpression of HMGN2 in A549 cells. This result further confirmed the cell adhesion assay data that HMGN2 knockdown increased $\alpha 5 \beta 1$ integrin activity by increasing talin expression. The activation of integrin leads to the connection of the $\alpha$ subunit to extracellular ligands and the $\beta$-subunit mediates the transfer signal from the outside to the inside of the cell, which affects the biological behavior of cell cytoskeletal rearrangement, adhesion, migration, proliferation and differentiation (32).

The conformation of the host cell cytoskeleton plays a crucial role in the process of bacterial internalization (33). Actin filaments are the basic component of the cytoskeleton that exist in two essential forms: monomeric (G-actin) and filamentous (F-actin). The F-actin filaments are highly dynamic structures, and their supermolecular organization is constantly modified according to cellular needs (34). Previous studies have suggested that the reorganization of the cytoskeleton is beneficial to bacterial internalization. Activated integrin transfers signal to downstream molecules and changes the actin polymerization state through integrin adaptor proteins $(27,35)$. FAK/Src as the integrin adaptor protein may bridge a number of downstream signaling pathways. Some previous studies have indicated that the FAK phosphorylation induces actin rearrangement in intestinal and reproductive tract epithelial cells $(36,37)$. Another study has reported that actin polymerization is induced by Src signaling in kidney epithelial cells (38). Therefore, we examined FAK/Src signaling in A549 cells following HMGN2 silencing. The results of western blot analysis revealed that knockdown of HMGN2 activated FAK/Src signaling. In addition, our microarray analysis showed that the expression of SCAP2 that is downstream of Src signaling, was also induced by HMGN2 knockdown in A549 cells (7). In this context, we suggest that FAK/Src signaling downstream of integrin activation was involved in the HMGN2-regulated internalization of bacteria.

Furthermore, F-actin fluorescence data showed that F-actin was more polymerized in the pulmonary epithelial cells than in the bronchial epithelial cells following HMGN2 depletion and subsequent bacterial infection. Collectively, we believe that the role of HMGN2 in the regulation of bacterial internalization by respiratory epithelial cells is physiologically and pathologically meaningful. As we have indicated, HMGN2 knockdown increased the rearrangement of actin filaments and bacterial internalization, and thus, the sensitivity to respiratory infections would also be increased in HMGN2-depleted organisms. Furthermore, it has been reported that HMGN2 expression is absent in adult animals (39). Moreover, it is well known that $K$. pneumoniae often occurs in the elderly (40); thus, determining whether HMGN2 plays a unique role in aging-related infectious diseases warrants further investigation.

In conclusion, our results indicate that the inhibition of HMGN2 in respiratory epithelial cells with HMGN2-specific
siRNA increased the internalization of $\mathrm{K}$. pneumoniae through the enhanced expression and activity of $\alpha 5 \beta 1$ integrin. The precise signaling pathway involved in this process is that of talin-mediated integrin activation, which led to the phosphorylation of FAK and Src, and further induced F-actin polymerization (Fig. 6D). From a therapeutic point of view, targeting HMGN2 may prove to be a valuable defense tool against pulmonary infection caused by $K$. pneumoniae, through the inhibition of the interactions and crosstalk between respiratory epithelial cells and $K$. pneumoniae.

\section{Acknowledgements}

The present study was supported, in whole or in part, by the National Natural Science Foundation of China (nos. 30470763, 81470931 and 31401188), and the Youth Foundation of Sichuan University (no. 2014SCU11042).

\section{References}

1. Körner U, Bustin M, Scheer U and Hock R: Developmental role of HMGN proteins in Xenopus laevis. Mech Dev 120: 1177-1192, 2003.

2. Mosevitsky MI, Novitskaya VA, Iogannsen MG and Zabezhinsky MA: Tissue specificity of nucleo-cytoplasmic distribution of HMG1 and HMG2 proteins and their probable functions. Eur J Biochem 185: 303-310, 1989.

3. Reeves R and Adair JE: Role of high mobility group (HMG) chromatin proteins in DNA repair. DNA Repair (Amst) 4: 926-938, 2005.

4. West KL, Castellini MA, Duncan MK and Bustin M: Chromosomal proteins HMGN3a and HMGN3b regulate the expression of glycine transporter 1. Mol Cell Biol 24: 3747-3756, 2004.

5. Belova GI, Postnikov YV, Furusawa T, Birger Y and Bustin M: Chromosomal protein HMGN1 enhances the heat shock-induced remodeling of Hsp70 chromatin. J Biol Chem 283: 8080-8088, 2008.

6. Zhu N and Hansen U: HMGN1 modulates estrogen-mediated transcriptional activation through interactions with specific DNA-binding transcription factors. Mol Cell Biol 27: 8859-8873, 2007.

7. Deng LX, Wu GX, Cao Y, Fan B, Gao X, Luo L and Huang N: The chromosomal protein HMGN2 mediates lipopolysaccharideinduced expression of $\beta$-defensins in A549 cells. FEBS J 278: 2152-2166, 2011.

8. Deng LX, Wu GX, Cao Y, Fan B, Gao X, Tang XH and Huang N: The chromosomal protein HMGN2 mediates the LPS-induced expression of $\beta$-defensins in mice. Inflammation 35: 456-473, 2012.

9. Feng Y, Huang N, Wu Q and Wang B: HMGN2: a novel antimicrobial effector molecule of human mononuclear leukocytes? J Leukoc Biol 78: 1136-1141, 2005.

10. Cao Y, Wu G, Fan B, Zheng F, Gao X, Liu N, Liu X and Huang N: High mobility group nucleosomal binding domain 2 protein protects bladder epithelial cells from Klebsiella pneumoniae invasion. Biol Pharm Bull 34: 1065-1071, 2011.

11. Wu G, Cao Y, Fan B, Zheng F, Gao X, Liu N, Liu X and Huang N: High-mobility group protein N2 (HMGN2) inhibited the internalization of Klebsiella pneumoniae into cultured bladder epithelial cells. Acta Biochim Biophys Sin (Shanghai) 43: 680-687, 2011.

12. Cossart P and Sansonetti PJ: Bacterial invasion: the paradigms of enteroinvasive pathogens. Science 304: 242-248, 2004.

13. Ulanova M, Gravelle S and Barnes R: The role of epithelial integrin receptors in recognition of pulmonary pathogens. J Innate Immun 1: 4-17, 2009.

14. Dingemans AM, van den Boogaart V, Vosse BA, van Suylen RJ, Griffioen AW and Thijssen VL: Integrin expression profiling identifies integrin alpha5 and beta 1 as prognostic factors in early stage non-small cell lung cancer. Mol Cancer 9: 152, 2010.

15. Sayeed A, Fedele C, Trerotola M, Ganguly KK and Languino LR: IGF-IR promotes prostate cancer growth by stabilizing $\alpha 5 \beta 1$ integrin protein levels. PLoS One 8: e76513, 2013. 
16. Docheva D, Padula D, Schieker $\mathrm{M}$ and Clausen-Schaumann $\mathrm{H}$ : Effect of collagen I and fibronectin on the adhesion, elasticity and cytoskeletal organization of prostate cancer cells. Biochem Biophys Res Commun 402: 361-366, 2010.

17. Takagi J, Erickson HP and Springer TA: C-terminal opening mimics 'inside-out' activation of integrin $\alpha 5 \beta 1$. Nat Struct Mol Biol 8: 412-416, 2001.

18. Calderwood DA, Zent R, Grant R, Rees DJG, Hynes RO and Ginsberg MH: The Talin head domain binds to integrin $\beta$ subunit cytoplasmic tails and regulates integrin activation. J Biol Chem 274: 28071-28074, 1999

19. Lafont $F$ and van der Goot FG: Bacterial invasion via lipid rafts. Cell Microbiol 7: 613-620, 2005.

20. Agarwal V, Ahl J, Riesbeck K and Blom AM: An alternative role of Clq in bacterial infections: facilitating Streptococcus pneumoniae adherence and invasion of host cells. J Immunol 191: 4235-4245, 2013.

21. March C, Moranta D, Regueiro V, Llobet E, Tomás A, Garmendia J and Bengoechea JA: Klebsiella pneumoniae outer membrane protein $\mathrm{A}$ is required to prevent the activation of airway epithelial cells. J Biol Chem 286: 9956-9967, 2011.

22. Mohan Nair MK and Venkitanarayanan K: Role of bacterial OmpA and host cytoskeleton in the invasion of human intestinal epithelial cells by Enterobacter sakazakii. Pediatr Res 62 : 664-669, 2007.

23. Alexander EH and Hudson MC: Factors influencing the internalization of Staphylococcus aureus and impacts on the course of infections in humans. Appl Microbiol Biotechnol 56: 361-366, 2001.

24. Cue D, Southern SO, Southern PJ, Prabhakar J, Lorelli W, Smallheer JM, Mousa SA and Cleary PP: A nonpeptide integrin antagonist can inhibit epithelial cell ingestion of Streptococcus pyogenes by blocking formation of integrin alpha 5 beta 1-fibronectin-M1 protein complexes. Proc Natl Acad Sci USA 97: 2858-2863, 2000.

25. Vinogradova O, Velyvis A, Velyviene A, Hu B, Haas T, Plow E and Qin J: A structural mechanism of integrin alpha(IIb)beta(3) 'inside-out' activation as regulated by its cytoplasmic face. Cell 110: 587-597, 2002.

26. Luo B-H, Carman CV and Springer TA: Structural basis of integrin regulation and signaling. Annu Rev Immunol 25: 619-647, 2007

27. Xiao T, Takagi J, Coller BS, Wang JH and Springer TA: Structural basis for allostery in integrins and binding to fibrinogen-mimetic therapeutics. Nature 432: 59-67, 2004
28. Tang $\mathrm{CH}$, Keng YT and Liu JF: HMGB-1 induces cell motility and $\alpha 5 \beta 1$ integrin expression in human chondrosarcoma cells. Cancer Lett 322: 98-106, 2012.

29. Johnson MS, Lu N, Denessiouk K, Heino J and Gullberg D: Integrins during evolution: evolutionary trees and model organisms. Biochim Biophys Acta 1788: 779-789, 2009.

30. Anderson LR, Owens TW and Naylor MJ: Structural and mechanical functions of integrins. Biophys Rev 6: 203-213, 2014

31. Anthis NJ, Wegener KL, Ye F, Kim C, Goult BT, Lowe ED, Vakonakis I, Bate N, Critchley DR, Ginsberg MH and Campbell ID: The structure of an integrin/talin complex reveals the basis of inside-out signal transduction. EMBO J 28: 3623-3632, 2009.

32. Margadant C, Monsuur HN, Norman JC and Sonnenberg A: Mechanisms of integrin activation and trafficking. Curr Opin Cell Biol 23: 607-614, 2011.

33. Campellone KG: Cytoskeleton-modulating effectors of enteropathogenic and enterohaemorrhagic Escherichia coli: Tir, EspFU and actin pedestal assembly. FEBS J 277: 2390-2402, 2010.

34. Navarro-Garcia F, Serapio-Palacios A, Ugalde-Silva P, Tapia-Pastrana $G$ and Chavez-Dueñas L: Actin cytoskeleton manipulation by effector proteins secreted by diarrheagenic Escherichia coli pathotypes. BioMed Res Int 2013: 374395, 2013.

35. Zhu J, Carman CV, Kim M, Shimaoka M, Springer TA and Luo BH: Requirement of $\alpha$ and $\beta$ subunit transmembrane helix separation for integrin outside-in signaling. Blood 110: 2475-2483, 2007.

36. Dia VP and Gonzalez de Mejia E: Lunasin potentiates the effect of oxaliplatin preventing outgrowth of colon cancer metastasis, binds to $\alpha 5 \beta 1$ integrin and suppresses FAK/ERK/NF- $\kappa B$ signaling. Cancer Lett 313: 167-180, 2011.

37. Siu MK, Wong CH, Xia W, Mruk DD, Lee WM and Cheng CY: The $\beta 1$-integrin-p-FAK-p130Cas-DOCK180-RhoA-vinculin is a novel regulatory protein complex at the apical ectoplasmic specialization in adult rat testes. Spermatogenesis 1: 73-86, 2011.

38. Pawlak G and Helfman DM: MEK mediates v-Src-induced disruption of the actin cytoskeleton via inactivation of the Rho-ROCK-LIM kinase pathway. J Biol Chem 277: 26927-26933, 2002.

39. Crippa MP, Nickol JM and Bustin M: Developmental changes in the expression of high mobility group chromosomal proteins. J Biol Chem 266: 2712-2714, 1991.

40. Fein AM: Pneumonia in the elderly: overview of diagnostic and therapeutic approaches. Clin Infect Dis 28: 726-729, 1999. 